\title{
An improved equation of state for air plasma simulations
}

\author{
F. Träuble, ${ }^{1, a)}$ S. T. Millmore, ${ }^{1, b)}$ and N. Nikiforakis ${ }^{1}$ \\ Cavendish Laboratory, University of Cambridge, Cambridge, United Kingdom
}

(Dated: 12 January 2021)

This work is concerned with the development of a novel, accurate equation of state for describing partially ionised air plasma in local thermodynamic equilibrium. One key application for this new equation of state is the simulation of lightning strike on aircraft. Due to the complexities of species ionisation and interaction, although phenomenological curve fitting of thermodynamic properties is possible, these curves are intractable for practical numerical simulation. The large difference in size of the parameters (many orders of magnitude) and complexity of the equations means they are not straightforward to invert for conversion between thermodynamic variables. The approach of this paper is to take an accurate 19-species phenomenological model, and use this to generate a tabulated data set. Coupled with a suitable interpolation procedure this offers an accurate and computationally efficient technique for simulating partially ionised air plasma. The equation of state is implemented within a multiphysics methodology which can solve for two-way coupling between a plasma arc and an elastoplastic material substrate. The implementation is validated against experimental results, both for a single material plasma, and an arc coupled to a substrate. It is demonstrated that accurate, oscillation-free thermodynamic profiles can be obtained, with good results even close to material surfaces.

\section{INTRODUCTION}

Numerical simulation of air plasma interacting with solid substrates provides insight into the physical mechanisms which lead to structural damage due to lightning strike, which is a key safety concern for aircraft ${ }^{1}$. The aluminium skin traditionally used for aircraft rapidly dissipates the energy input from the lightning strike, due to its high electrical and thermal conductivity. With the current trends towards lightweight composite skins, with lower conductivity, there is greater energy input local to the lightning attachment point. To accurately understand the physical effects at this attachment, an accurate description of the ionisation processes within the plasma arc is essential. This requires an equation of state (EoS) which can account for the complex thermodynamic and electromagnetic effects arising from partially ionised material. The multiple atomic species comprising an air plasma are each governed by different characteristic ionisation energies, governing their initial emergence and further ionisation. This leads to a non-linear relationship between intensive thermodynamic variables within the EoS for an air plasma. Computation of these quantities, which on a continuum scale model of a plasma are local physical properties, and are independent of system size, must still take into account transport properties arising from molecular interactions between the different species present.

It is a key challenge in developing an EoS for air plasma to balance accuracy with computational efficiency. One of the simplest techniques is to use a surrogate ideal gas model with an adiabatic index appropriate for much of the ionised regime, for example Ekici et al ${ }^{[2}$ use a an effective adiabatic exponent of $\gamma=1.16$. In a lightning arc simulation, for which there is the transition of unionised air to a plasma, an ideal gas model has limited validity. An improved model was developed by Plooste ${ }^{[3}$ and has been applied in several plasma

\footnotetext{
a) Also at Max Planck Institute for Intelligent Systems, Tübingen, Germany

${ }^{b)}$ Electronic mail: stm31@ cam.ac.uk
}

discharge models. It is a simplified equation of state for a surrogate diatomic gas, which takes into account dissociation effects. However, due to the different dissociation energies for $\mathrm{N}_{2}$ and $\mathrm{O}_{2}$, key components of an air plasma, and the fact that nitric oxide $(N O)$ is not considered, this approach is limited to lower temperature mixtures, below 9,000K ${ }^{4}$. Villa et al! ${ }^{5}$ developed an EoS which models 11 of the most common species within an air plasma, allowing for single ionisation of $\mathrm{N}_{2}$ and $\mathrm{O}_{2}$, as well as the dissociated atoms. Each species is treated as an ideal gas which are then combined as a generalised ideal gas mixture. They start from the work of Mottura et al 6 who assume a simplified heat capacity of the form $C_{v}(T)$ with $T$ being the temperature, neglecting effects from a variation in pressure. The equation of state for each species assumes a linear relation between the specific heat contribution and species' dependent formation heats. To fully describe a given set of thermodynamic data, a system of coupled linear equations must be solved. In order to maintain computational efficiency, Villa et al. tabulated the thermodynamic data for given pairs of density and specific internal energy, and interpolate values between these points. This tabulated form allows for a complex description of the plasma composition, though, in common with other equations of state available in the open literature, it does not consider plasma-specific effects arising from electromagnetic interaction on microscopic scales.

In this work, an improved equation of state is developed based on an accurate air plasma model covering a wide range of variables from the theoretical model of D'Angola et al.78? In section 2 the thermodynamic theory of air plasma, upon which the equation of state is based, is detailed. Additionally, a mathematical formulation for describing the governing equations of motion for an air plasma, which will be used to validate the improved EoS, are given, using the model developed by Millmore and Nikiforakis 9 . The generation of the new EoS is detailed in section 3 and section 4 presents validation of the improved EoS within a state-of-the-art multiphysics multimaterial lightning code. It is demonstrated that an accurate EoS is required to model the arc attachment point 
to a substrate, and thus is essential in order to gain a deeper insight into this highly complex and nonlinear phenomenon. Conclusions and further work are presented in section 5.

\section{THEORY AND MATHEMATICAL FORMULATION}

\section{A. Air plasma model}

Atmospheric air is a mixture of many different particle species which, over the temperature and pressure range experienced in a lightning strike, will interact in a complex mannel ${ }^{10}$. The composition of dry air per volume is primarily made up of $78.084 \%$ nitrogen, $20.946 \%$ oxygen, $0.934 \%$ Argon and $0.036 \%$ carbon dioxide. Additional species such as other noble gases occur not more than a few parts per million particles. Under realistic conditions, there is a variable amount of water vapour present in the air ${ }^{11 / 12}$.

An appropriately chosen theoretical model of air plasma has to cover the physics between the limits of unionised lowtemperature air and highly ionised air plasma. The generation of the EoS presented here is based on an accurate theoretical model for a 19-species air plasma model developed by Capitelli, Colonna, D'Angola and others in $7 / 8|10| 13-15$. This air plasma model considers the following 19 air species:

$$
\begin{gathered}
\mathrm{N}_{2}, \mathrm{~N}_{2}^{+}, \mathrm{N}, \mathrm{N}^{+}, \mathrm{N}^{++}, \mathrm{N}^{+++}, \mathrm{N}^{++++} \\
\mathrm{O}_{2}, \mathrm{O}_{2}^{+}, \mathrm{O}_{2}^{-}, \mathrm{O}, \mathrm{O}^{-}, \mathrm{O}^{+}, \mathrm{O}^{++}, \mathrm{O}^{+++}, \mathrm{O}^{++++} \\
\mathrm{NO}, \mathrm{NO}^{+}, e^{-}
\end{gathered}
$$

Molecular species with more than two atoms are not considered, as they only occur in low quantities, so their effect on the application presented here is negligible, and the theory for excited energy levels of these species is complex. The chemical processes among the different species are assumed to be in detailed balance at all times with the only exception being radiation processes, which involve the emission and absorption of photons. This assumption is valid as long as molecular timescales are significantly smaller than fluid and gas flow timescales; this is satisfied under the thermodynamic conditions of interest. In this case, a unique temperature and pressure can be assigned to a given composition. As such intensive variables are inhomogeneous in time and space for the applications of interest in this paper, the concept of local thermodynamic equilibrium (LTE) needs to be defined. LTE requires that the particles' energies in a local neighbourhood obey Maxwell-Boltzmann distributions, so that a local temperature and pressure can be defined. This is not always perfectly justified, but work from Haidar ${ }^{16}$ indicates that it is sufficiently accurate to predict the expected behaviour in a lightning attachment.

\section{Thermodynamics}

In dynamical equilibrium, every reaction is governed by a law of mass action, taking the form

$$
K_{r}^{P}(T)=\prod_{s=1}^{N_{\text {total }}} P_{s}^{v_{r, s}} \quad r=1,2, \ldots, N_{R}
$$

where $K_{r}^{P}(T)$ is a temperature dependent equilibrium constant of the $r^{t h}$ reaction and $P_{S}$ is the partial pressure of the $s^{t h}$ species. $N_{\text {total }}$ is the number of considered species and $v_{r, s}$ the stoichiometric coefficients of the $s^{t h}$ species in the $r^{\text {th }}$ reaction. The total pressure of a gas mixture is then given by Dalton's law

$$
P=\sum_{s=1}^{N_{\text {total }}} P_{s}+P_{D H}
$$

where the correction term $P_{D H}$, derived from the DebyeHückel theory, considers additional effects for ionised gas mixtures, with further details below. Every species is partially approximated by $P_{s}=n_{s} k_{B} T$ with $n_{s}$ the particle number density of the $s^{\text {th }}$ species, $k_{B}$ the Boltzmann constant and $T$ the temperature. The particles are modelled as dimensionless hard spheres and virial corrections are neglected. This is justified as they are usually only important for $T<300 \mathrm{~K}$ with pressure of $P \approx 100$ atm $17-19$, i.e. unrealistic conditions for the arc channel plasma and surrounding air.

For given pressure and temperature, the system of nonlinear equations arising from (1) and (2) determines the exact composition of all the species present in the air model. A further condition is given by the conservation of the predetermined total mass of each atomic species, adding two additional constraints to the 17 equations to close the system. It is challenging to solve such a nonlinear system numerically, since equilibrium constants differ by many orders of magnitude. The composition used for the numerical fits in ${ }^{7 / 8}$ is based on an accurate hierarchical algorithm for solving the equilibrium of a given reactive mixture 8 .

Once the composition $\left\{n_{s}\right\}_{s=1, \ldots, N_{\text {total }}}$ is known, the thermodynamic potentials are calculated using the respective partition function for each species $7 / 13$. Due to the formation of a plasma potential in ionised gases, and plasma in general, the thermodynamic properties are modified when compared to an ideal gas. The theory and framework to consider such effects were developed in the Debye-Hückel theory 20. This introduces a cut-off energy, making the sum concerning the internal partition functions finite and further correction terms for the pressure and potential $\sqrt{13}$. Finally, the heat capacities are given by

$$
\begin{aligned}
C_{P} & =\left(\frac{\partial H}{\partial T}\right)_{P} \\
C_{V} & =\left(\frac{\partial U}{\partial T}\right)_{V}
\end{aligned}
$$

with $H$ being the enthalpy and $U$ the internal energy. 


\section{Transport properties}

In the presence of gradients in the spatial distribution of thermodynamic quantities, transport effects emerge which act to reduce these gradients. Each thermodynamic behaviour has a different responsible transport mechanism. Ordinary diffusion is associated with a concentration difference and thermal diffusion connected to a gradient in temperature. Viscosity is the mechanism describing the transfer of momentum due to velocity gradients, while thermal conductivity contains effects that lead to transport in thermal energy such as thermal gradients, chemical reactions or redistributions of internal degrees of freedom. Electrical conductivity is related to the transport of charged particles due to differences in the electric potential. At energies relevant to air plasmas within this work, it is justified to assume that the transport coefficients of electrons and heavy atoms or ions decouple from each other ${ }^{21}$. $\mathrm{Nu}-$ merical fits regarding the transport coefficients in ${ }^{7 / 8}$ have been calculated using third-order approximated coefficients derived from the Chapman-Enskog theory ${ }^{[/ 22}$. The Chapman-Enskog theory provides a set of equations for calculating the transport properties of a multi-species gas mixture under the assumption that thermodynamic states can be described in LTE. Its starting point is the Boltzmann equation formulation and it assumes some justified microscopic models for the binary collision term 23 . The main approach of the theory represents a Chapman-Enskog expansion where the probability density function is expanded perturbatively in a small parameter $\varepsilon$. This will result in a general set of Navier-Stokes equations for this system, including terms for transport coefficients. A detailed derivation can be found in Capitelli et al ${ }^{10}$, and this derivation provides expressions for characteristic transport coefficients to an arbitrary degree of order $\xi$. In this work, the transport coefficients of interest are electrical and thermal conductivity 21 . Corresponding collision integrals, as calculated by D'Angola et al., take into account a variety of speciesdepended interaction approximations.

\section{B. Mathematical formulation of a plasma discharge}

To model the dynamics of a plasma discharge for lightning studies, a magnetohydrodynamic formulation is used. This assumes the plasma is modelled as a single fluid with a corresponding equation of state that considers thermodynamics of interacting species; this is appropriate for an air mixture in LTE. The governing equations are

$$
\begin{aligned}
\frac{\partial}{\partial t} \rho+\nabla \cdot(\rho \boldsymbol{u}) & =0 \\
\frac{\partial}{\partial t}(\rho \boldsymbol{u})+\nabla \cdot(\rho \boldsymbol{u} \otimes \boldsymbol{u}+P \boldsymbol{I}) & =\boldsymbol{J} \times \boldsymbol{B} \\
\frac{\partial}{\partial t} E+\nabla \cdot(\boldsymbol{u}(E+P)) & =\boldsymbol{u} \cdot(\boldsymbol{J} \times \boldsymbol{B})+\rho_{\text {res }}|\boldsymbol{J}|^{2}-S_{r} \\
\nabla^{2} \boldsymbol{A} & =-\mu_{0} \boldsymbol{J}
\end{aligned}
$$

with density, $\rho$, velocity $u$, total energy $E=\rho e+\frac{1}{2} \rho u^{2}$, magnetic potential, $\boldsymbol{A}$, magnetic field $\boldsymbol{B}=\nabla \times \boldsymbol{A}$, current density, $\boldsymbol{J}$, electrical resistivity $\rho_{\text {res }}=1 / \sigma$, electrical conductivity $\sigma$, permeability of free space $\mu_{0}$, and a source term governing radiative losses, $S_{r}$. As the system is underdetermined ( 9 unknowns in 8 equations), one further equation is needed for closure. This is provided by an equation of state for air plasma, providing the mathematical relation between specific internal energy $(e)$, pressure $(P)$ and mass density, in the form $e=e(\rho, P)$. Within this framework, a cylindrically axisymmetric geometry is considered, with the arc connection at the centre of the domain, which is sufficient to capture the bulk behaviour of the arc-substrate interaction 24 . The system, which is representative of a typical set up in lightningprotection laboratory experiments, is schematically shown in Figure 1. Current profiles representative of those used in labo-

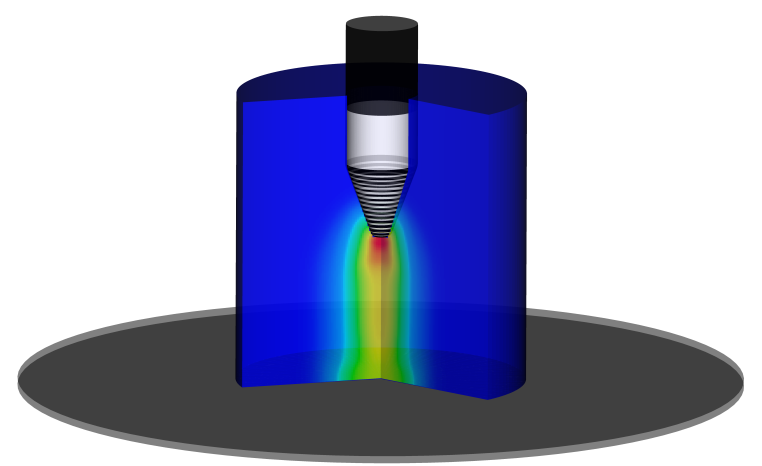

FIG. 1. Schematic showing the axisymmetric plasma discharge setup. A plasma arc is generated at the electrode at the top of the image, and connects to the substrate beneath it. A cut-through of the air/plasma region is shown, illustrating the arc; the plasma does occupy the full domain, to the edge of the substrate. Beneath the substrate is a subsequent air region (not shown), an approximate twodimensional representation of this setup is shown in Figure 2

ratory lightning testing are included within the model through the current density. Two different models are studied: a simplified 1D model and a more realistic axisymmetric model.

Within the 1D model, radial arc profiles are reproduced by assuming translational invariance along the $\mathrm{z}$-coordinate. Consequently, the current density has a component only in the $z$-direction and is approximated by a Gaussian profile as suggested in 5

$$
\boldsymbol{J}(r, t)=-\frac{I(t)}{\pi r_{0}^{2}} e^{-\left(r / r_{0}\right)^{2}} \boldsymbol{e}_{z}
$$

where $r_{0}$ is the characteristic length scale of the plasma arc channel radius and the total current $I(t)$ is a given input function. By assuming such a predetermined profile, the resulting magnetic field is not coupled to the fluid evolution itself.

Within the axisymmetric model, the geometry of an electrode is explicitly included and the current density is computed dynamically. Hence, this approach is more accurate as it 
calculates the magnetic field in a plasma arc self-consistently since it is coupled with the evolution of the air plasma. It also allows for interaction with the boundaries to affect the current profile. The current density is connected to the electric field $\boldsymbol{E}$ or its corresponding potential $\phi$ through

$$
\boldsymbol{J}=\sigma \boldsymbol{E}=-\sigma \nabla \phi
$$

and due to the conservation of charge, it holds $\nabla \cdot \boldsymbol{J}=0$. As a consequence, elliptic PDEs for $\phi$ and $\boldsymbol{A}$ have to be solved, given by

$$
\begin{aligned}
\nabla \cdot(\sigma \nabla \phi) & =0 \\
\nabla^{2} \boldsymbol{A} & =\mu_{0} \sigma \nabla \phi
\end{aligned}
$$

which represent some form of generalised Poisson's equations on the domain. Figure 2 shows the domain and its ge-

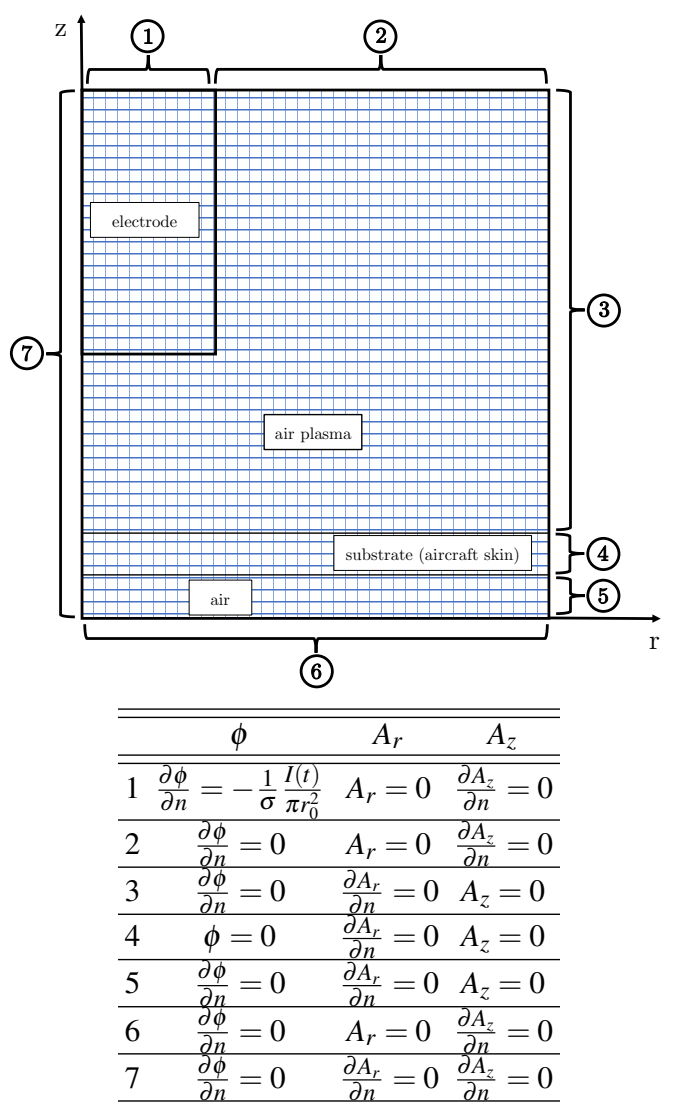

FIG. 2. Geometry of the computational domain in the 2D model (left) including boundary conditions for the electromagnetic potentials (right).

ometry for the 2D axisymmetric model. Applied boundary conditions for the electromagnetic potentials are summarised in the table beneath the diagram. If an aluminium panel is used as the aeronautical substrate, an electrical conductivity of $\sigma=3.2 \times 10^{7} \mathrm{Sm}^{-1}$ is used, i.e. higher than typical air plasma conductivity within the arc channel. Alternatively, this substrate will be modelled as a carbon fibre composite material. A complete implementation of an anisotropic equation of state for carbon fibre composites is beyond the scope of this work. Instead, an approximation to CFRP is used, following 9 , which defines the material as a isotropic material, which can be employed to look at behaviour either perpendicular or parallel to the CFRP weave. This material differs from aluminium in electrical conductivity $\left(\sigma=1.6 \times 10^{4} \mathrm{Sm}^{-1}\right) \sqrt{25}$ and mass density $\left(\rho=1538 \mathrm{~kg} / \mathrm{m}^{3}\right)$.

The model described above is implemented within a more extensive simulation code. This includes explicit thermomechanical coupling with various aircraft layer configurations in a full multimaterial model 9 . This uses the Riemann Ghost Fluid Method (GFM) to accurately model the multimaterial boundary conditions, i.e. propagation and reflection of shock waves across a multimaterial interface. The location of the interface is tracked by a level set function. The original GFM

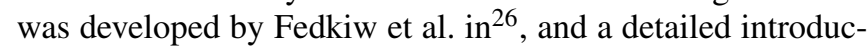
tion to Riemann GFM is given in the work of Sambasivan et al.27. Different materials are evolved independently from each other on their respective domains, and a unique equation of state can be assigned to each material. In this code, the elastoplastic substrate and electrode are described using the Eulerian framework as presented by Schoch et al. ${ }^{28}$ and Michael et al.29, based on the formulation of Godunov and Romenskil 30 . Plasticity effects are incorporated following the work of Miller and Collela ${ }^{31}$. Details are discussed in Millmore and Nikiforakis 9 , which validate the equations from the previous section.

\section{METHODOLOGY}

\section{A. Generating data for an improved equation of state}

D'Angola et al ${ }^{718}$ calculated a wide range of thermodynamic and transport quantities for given temperature and pressure (mixture composition, molar mass, enthalpy, heat capacities, electrical and thermal conductivity), based on the air plasma model described in section II A. Therewith an improved equation of state for a 19-species air plasma model was developed. The equation of state is inserted into the plasma discharge model from section II B by the help of a tabular database, denoted by EOS19. In principle, EOS19 can be used by any simulation code that attempts to model air plasma applications in the future. Every data point in the database generated contains the following quantities:

- mass density $\rho_{i}$

- pressure $P_{i}$

- specific internal energy $e_{i}$

- temperature $T_{i}$

- speed of sound $c_{i}$

- adiabatic index $\gamma_{i}$

- molar fractions of each species $\chi_{i}$

- electrical conductivity $\sigma_{i}$ 
- thermal conductivity $\kappa_{i}$

The actual relation for the equation of state is then given through discrete tuples of the form $\left(\rho_{i}, P_{i}, e_{i}\right)$ with $e\left(\rho_{i}, P_{i}\right)=e_{i}$. To generate this data the remaining quantities not provided as analytical fits in ${ }^{7 / 8}$ need to be calculated. The mass density of air plasma $\rho$ is calculated with the help of the mean molar mass $\bar{M}$ via the formula

$$
\rho=\frac{P}{R T} \bar{M}
$$

with $R$ being the gas constant. Specific internal energy is derived from the specific enthalpy with the help of a Legendre transformation according to

$$
e=h-\frac{P}{\rho}
$$

Although negligible in this model, thermal conductivity from analytical fits in ${ }^{7 / 8}$ is included in the database, such that it can be used to considering associated effects in the future. Finally, the adiabatic index and corresponding speed of sound, $a$, for air plasma as suggested in ${ }^{1332}$ are calculated as

$$
\begin{aligned}
& \gamma=\frac{C_{P}}{C_{V}} \frac{\rho}{P}\left(\frac{\partial P}{\partial \rho}\right)_{T} \\
& a=\sqrt{\gamma \frac{P}{\rho}}
\end{aligned}
$$

with the heat capacities defined in equations (3) and (4).

For typical choices of conserved variables, it is desirable to generate the thermodynamic EoS quantities for given pairs of density and pressure, instead of temperature and pressure. Therefore, the temperature has to be obtained inversely for given density and pressure in the form $T(\rho, P)$ with the help of the known relation in 13

$$
\rho\left(P_{i}, T_{i}\right)=\rho_{i}
$$

where the tuple $\left(T_{i}, P_{i}, \rho_{i}\right)$ represents a thermodynamical state. Finding the solution for $T_{i}$ in 17 reduces to a root-finding problem, and a simple bisection method is applied. It is not important to have an efficient algorithm here since the tabulated data is generated only once. However, even with a more advanced method, this root-finding process is timeconsuming, which is the reason for creating a tabulated database instead of calculating parameters dynamically during simulation. From this data, the equation of state is interpolated. The layout of EOS19 follows the SESAME Database standard for equations of state as introduced at Los Alamos National Lab 33 . It contains data points ranging from $0.001 \mathrm{~kg} / \mathrm{m}^{3}$ to $10 \mathrm{~kg} / \mathrm{m}^{3}$ in mass density and $0.01 \mathrm{~atm}$ to $180 \mathrm{~atm}$ in pressure. Interpolation from this database was achieved by a binary search algorithm.

\section{B. Numerical approach for solving the plasma discharge}

For each computational time-step $\Delta t$, the system of equations $(5)-(8)$ is evolved in four steps, following the approach in 5 .

Step 1 The Poisson's equation in $(8)$ for the magnetic vector potential $\boldsymbol{A}$ is solved using the Thomas-Algorithm in $1 \mathrm{D}$ and the finite element solver framework FEniCS ${ }^{34}$ in axisymmetry. Both algorithms are an appropriate choice regarding accuracy and efficiency, however any suitable solver could be used. In axisymmetry, the magnetic vector potential has the non-zero components $A_{z}(r, z)$ and $A_{r}(r, z)$. The corresponding magnetic field, pointing in the poloidal direction, is then given by

$$
B_{\phi}=(\nabla \times \boldsymbol{A})_{\phi}=\frac{\partial A_{r}}{\partial z}-\frac{\partial A_{z}}{\partial r}
$$

with $A_{r}=0$ in the $1 \mathrm{D}$ model.

Step 2 The homogeneous part of the Euler problem in (5) - (7), given by

$$
\begin{aligned}
\frac{\partial}{\partial t} \rho+\nabla \cdot(\rho \boldsymbol{u}) & =0 \\
\frac{\partial}{\partial t}(\rho \boldsymbol{u})+\nabla \cdot(\rho \boldsymbol{u} \otimes \boldsymbol{u}+P \boldsymbol{I}) & =0 \\
\frac{\partial}{\partial t} E+\nabla \cdot(\boldsymbol{u}(E+P)) & =0
\end{aligned}
$$

is solved using high-order shock-caputuring schemes. In this work, the second-order finite volume method SLIC (Slope LImiter Centred) is used 35 .

Step 3 To incorporate Joule heating and radiation source terms in the energy balance equation (7), an ordinary differential equation of the form

$$
\frac{d E}{d t}=\rho_{\text {res }}|\boldsymbol{J}|^{2}-S_{r}
$$

has to be solved, with details regarding the implementation of the radiation model given in 536 .

Step 4 To include Lorentz force effects, the ODEs given by

$$
\begin{aligned}
\frac{d}{d t}(\rho \boldsymbol{u}) & =\boldsymbol{J} \times \boldsymbol{B} \\
\frac{d E}{d t} & =\boldsymbol{u} \cdot(\boldsymbol{J} \times \boldsymbol{B})
\end{aligned}
$$

are solved by using a standard second-order ODE solver with two half-time step updates that conserves the energy and momentum 5 .

\section{RESULTS}

In this section, data from the improved, newly generated equation of state is shown together with its implementation in the $1 \mathrm{D}$ and axisymmetric plasma models. 


\section{EOS19 data}

The specific internal energy generated by EOS19 for a given mass density and pressure is shown in Fig. 3. These
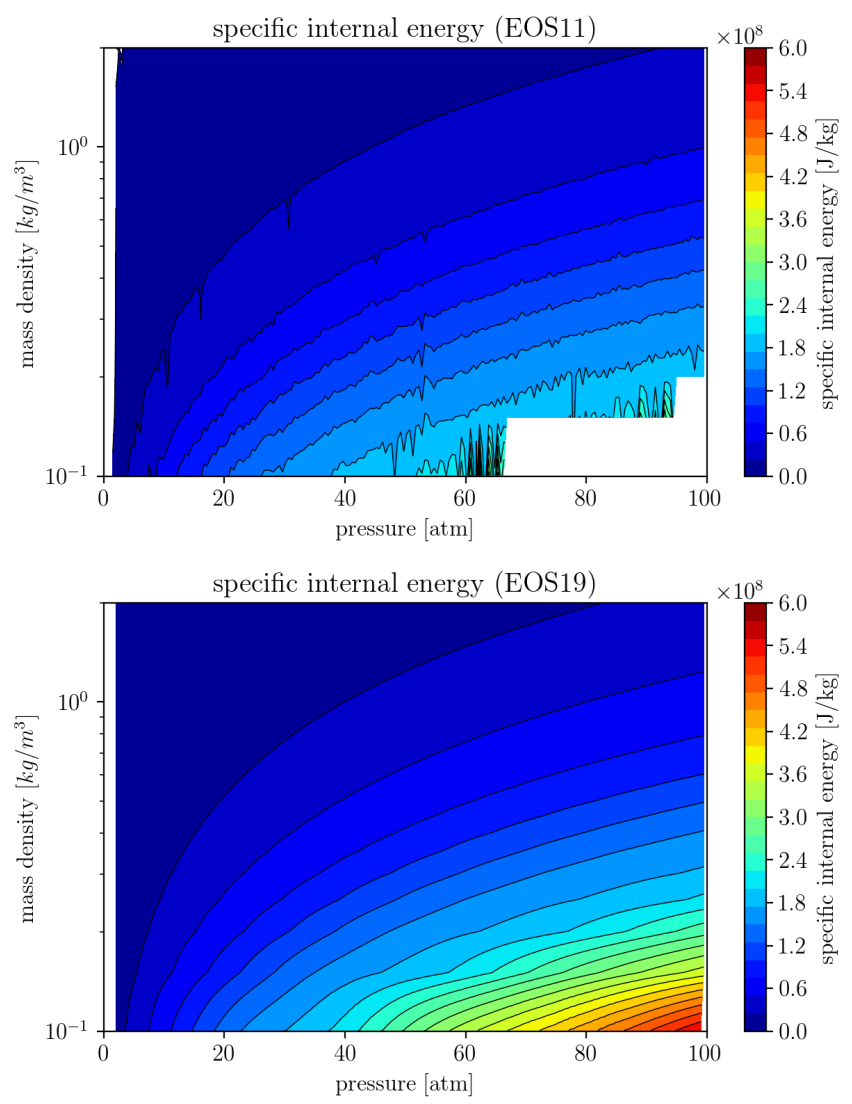

FIG. 3. Heat map of the specific internal energy for EOS11 (top) and EOS19 (bottom)

values are compared to the publicly available data of the equation of state developed by Villa, denoted as EOS11 5 [36. As there are no classical phase transitions observed in air plasmas, a physically accurate description requires smooth contour lines in any physical variable. Qualitatively, both equations of state show similar behaviour with smooth contours in EOS19. However, there are accuracy issues in EOS11, which generate unphysical unsteady features at low densities and high pressure as will be shown shortly. The missing region in EOS11 is due to a lack of sufficient data points for reliable interpolation.

\section{D Model}

Simulations in 1D were calculated on a radial domain with $r \in[0,0.2 \mathrm{~m}]$, with initial data is chosen to represent a realistic air state with mass density $\rho=1.225 \mathrm{~kg} / \mathrm{m}^{3}$, velocity $u=0 \mathrm{~m} / \mathrm{s}$ and pressure $P(r)=P_{0}+P_{1}(r)$ with $P_{0}=101,325 \mathrm{~Pa}$. Initially, the centre of the domain is preheated, as a nonvanishing electrical conductivity is needed to further heat the plasma sufficiently via the Joule heating to solve (10). This preheating condition is imposed by an added a Gaussian pressure profile $P_{1}=2 \times 10^{6} \mathrm{~Pa} \cdot \exp \left(-\left(r / r_{0}\right)^{2}\right)$ with $r_{0}=2 \mathrm{~cm}$, which results in an initial Gaussian temperature profile with $T_{\text {peak }} \approx 5,000 \mathrm{~K}$ in the centre of the arc. Radial profiles with the oscillatory component $V$ current are shown in Figure 4 for
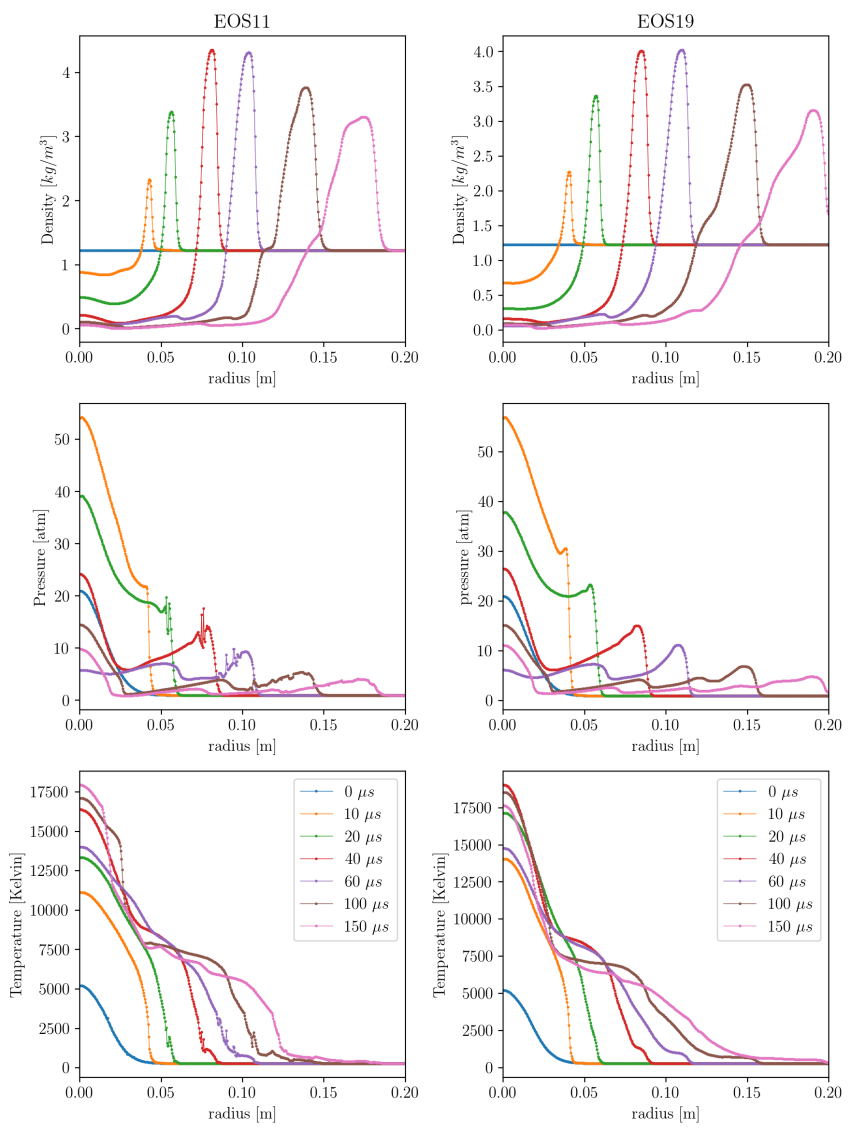

FIG. 4. Radial profiles $(N=500)$ for a component $V$ current for EOS11 (left) and the new EOS19 (right). In both cases, the results qualitatively agree, however, the oscillations visiible at about 2,000 K in the EOS11 results do not appear when using EOS19.

EOS19 and EOS11 in comparison. The mathematical form of a component $\mathrm{V}$ current profile, and others applied in this work, are detailed in the Table I] Both models show similar behaviour, within the first $10 \mu \mathrm{s}$, the plasma arc channel heats up quickly, and the pressure increases rapidly reaching peak values of $P_{\text {peak }}=55 \mathrm{~atm}$ for EOS19 and almost $P_{\text {peak }}=60 \mathrm{~atm}$ for EOS11. The formation of a radially expanding shock wave is observed, which leaves a low-density region in the centre of the arc channel. The speed of the shock wave is faster for EOS19, this difference is likely to be associated with EOS11 showing the incorrect internal energy at low temperatures. As the shock travels outwards, it undergoes geometric expansion, hence reduces in magnitude. At the centre of the arc, pressure and temperature show pulsed behaviour with time, which is a result of the oscillatory current profile, and this generates further pressure. The temperature in the centre remains between $12,500 \mathrm{~K}$ and $20,000 \mathrm{~K}$ whilst current input remains 


\begin{tabular}{|c|c|c|}
\hline Component & $D$ & $V$ (Villa) \\
\hline \hline$I(t)$ & $I_{0}\left(e^{-\alpha t}-e^{-\beta t}\right)\left(1-e^{-\gamma t}\right)^{2}$ & $I_{0} e^{-\alpha t} \sin (\beta t)$ \\
\hline$I_{0}[\mathrm{~A}]$ & 106,405 & 218,000 \\
\hline$\alpha\left[s^{-1}\right]$ & 22,708 & $4,137.95$ \\
\hline$\beta\left[s^{-1}\right]$ & $1,294,530$ & 114,866 \\
\hline$\gamma\left[s^{-1}\right]$ & $10,847,100$ & - \\
\hline time to peak $[\mu s]$ & 3.18 & 13.7 \\
\hline time to half-peak $[\mu s]$ & 34.5 & $41.1\left(2^{n d}\right.$ peak $)$ \\
\hline
\end{tabular}

TABLE I. Current components applied in simulations lightning strike testing. Component $D$ corresponds toa a single high-amplitude pulse with decreasing peak current as defined in 37? Additionally, a component $V$ current profile has been used in $\left[\frac{5}{5}\right.$ and reflects a high-amplitude oscillatory exponentially decaying current profile.

high. EOS11 displays oscillatory spikes in the pressure profile behind the leading shock wave; similar oscillations can be seen in the corresponding temperature profiles. These features occur in a temperature range between $T<1,000 \mathrm{~K}$ and $T<3,500 \mathrm{~K}$, corresponding to the region in which EOS 11 shows an unphysical concentration of of ionised species. In contrast, both temperature and pressure profiles for EOS19 are smooth everywhere indicating an improvement to the accuracy of the results. This demonstrates the importance of an accurate representation of the ionisation process in obtaining accurate behaviour within an air plasma.

Validation of the model presented in Section II A follows the approach of Villa et al 5 , where pressure loading due to the expansion of a cylindrical plasma arc is recorded. Specifically, in their experimental set up, they drilled three holes into the substrate at radial distances $5 \mathrm{~cm}, 10 \mathrm{~cm}$ and $15 \mathrm{~cm}$ from the arc attachment point. These holes were connected by a $25 \mathrm{~cm}$ tube to pressure transducers which recorded the effects of the passing shock wave and subsequent post-shock behaviour. Villa et al. compared these experimental results with their numerical simulations using a one-dimensional approximation for the tube; here this test is used to validate data for the equation of state and plasma model. Results for the pressure at the three locations using the model presented here, both with EOS11 and EOS19, are shown in Figure 5. The two models show qualitatively similar behaviour, although EOS19 predicts a higher pressure peak than EOS11 upon arrival of the initial wave. However, the subsequent pressure decrease after this peak is then more consistent with experimental measurements from Villa ${ }^{5}$ for EOS19. The finite width of the tubes is not modelled in the one-dimensional simulation of the behaviour, hence it is likely that the experimental pressures near the peak are slightly flattened due to geometric effects not captured by the numerical models.

\section{Multimaterial lightning code}

Having validated EOS19 in isolation, it is now used within the multimaterial model introduced in Millmore and Nikiforakis 9 . Using this, it is possible to investigate the effect
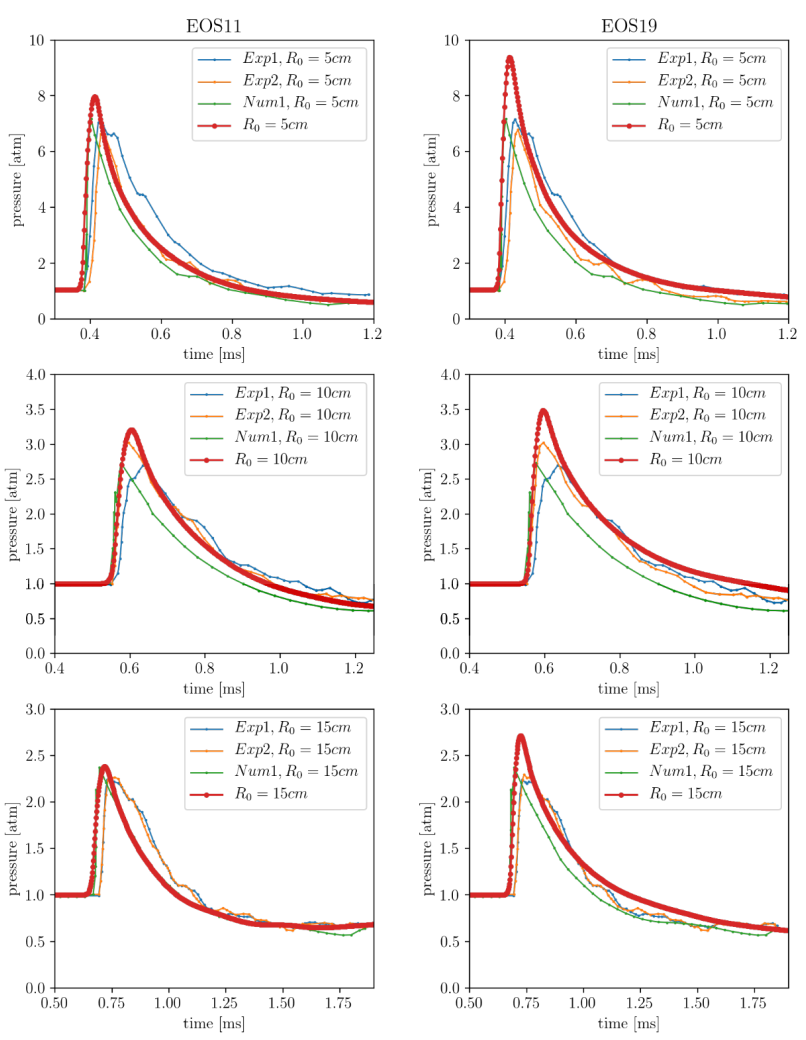

FIG. 5. Pressures calculated at the end of transducer tubes of length $L=15 \mathrm{~cm}$ being $5 \mathrm{~cm}, 10 \mathrm{~cm}$ and $15 \mathrm{~cm}$ away from the centre. Results in comparison for EOS11 (left) and EOS19 (right) with experimental ('Exp1' and 'Exp2') and numerical ('Num1') results from Villa et al $[5$.

of the EoS on simulations of arc attachment, both qualitatively and quantitatively.

The experiments of Martins ${ }^{24}$ provide imaging of the arc evolution attaching to various substrates, as well as measurements of the expansion of both the shock wave generated by the attachment, and of the arc itself. These tests use a component $D$ current, as defined in table I] and a simulation in line with a typical laboratory set up is considered, a conical electrode geometry with a flattened tip of radius $2.3 \mathrm{~mm}$ is used. The substrate (see setup in Figure 2) has a thickness of $\delta=2 \mathrm{~mm}$ and is modelled as an elastoplastic solid, as described in Millmore and Nikiforakis 9 .

For all tests, initial data in the air plasma, outside of the arc, is $\rho=1.225 \mathrm{~kg} / \mathrm{m}^{3}, \boldsymbol{u}=0 \mathrm{~m} / \mathrm{s}$ and $P_{0}=101,325 \mathrm{~Pa}$. For the arc channel to be initially conductive, the air is preheated to $8,063 \mathrm{~K}$, within a thin region of radius $r_{0}=2 \mathrm{~mm}$, for the full height between the electrode and the substrate. This value corresponds to $P=40 \times 101,325 \mathrm{~Pa}$ and $\sigma \approx 312 \mathrm{Sm}^{-1}$. The domain beneath the substrate is air, modelled as an ideal gas with $\gamma=1.4$.

Martins considers arc attachment to two materials, aluminium and CFRP; here results are presented using the techniques described in section II B, using a quasi-isotropic model for CFRP, which is henceforth referred to as a low- 
conductivity substrate.

Figure 6 shows illustrative temperature and pressure pro-
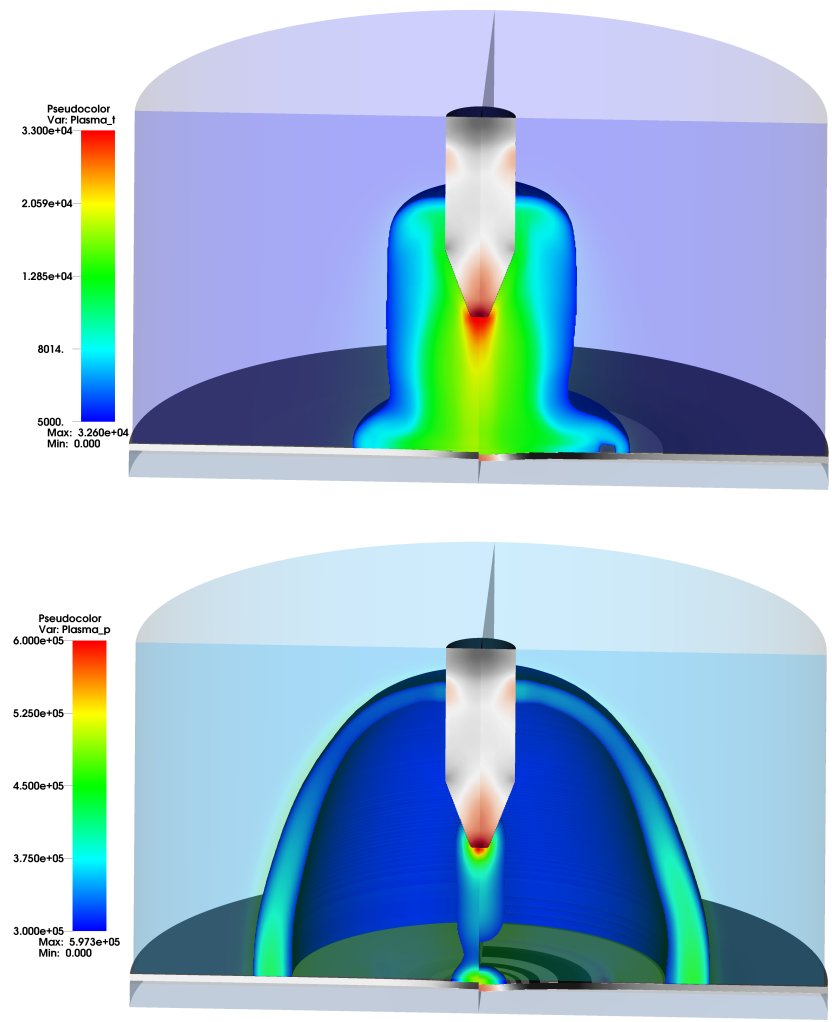

FIG. 6. Results for the plasma arc interacting with an aluminium panel (left half) and CFC panel (right half) at $t=50 \mu \mathrm{s}$. The top plot shows temperature and the bottom plot shows pressure.

files for the arc attachment to both substrates at $t=50 \mu$ s using EOS19.

In order to understand the difference between the two equations of state for arc attachment, the initial data described above is simulated with both EOS11 and EOS19. Results are shown for arc attachment to an aluminium substrate at two time instances, $t=3.18 \mu \mathrm{s}$ and $t=25 \mu \mathrm{s}$, for pressure and temperature in Figures 7 and 8 respectively. The first of the two times corresponds to the peak current input time and there are similar pressure profiles for both EOS11 and EOS19. It is noted that EOS11 results in a lower pressure directly above the substrate than for EOS19, whilst the pressure within the shock wave appears to be higher, indicated by the contour plotted at $P=4 \times 10^{6} \mathrm{~Pa}$. This higher pressure in the peak is likely a result of the oscillatory behaviour within the shock wave shown in Figure 4. However, there are clear differences in the temperature profiles between the two equations of state, even at this early time. EOS11 displays unphysical hot spot-like domains, with temperatures of up to $150,000 \mathrm{~K}$, close to electrode and substrate, most likely due to incorrect thermodynamic states obtained for coupling the materials boundaries in EOS11. Such hot spots are not observed for for EOS19, where temperatures do not exceed $67,500 \mathrm{~K}$, and nor are they evident in the experimental work
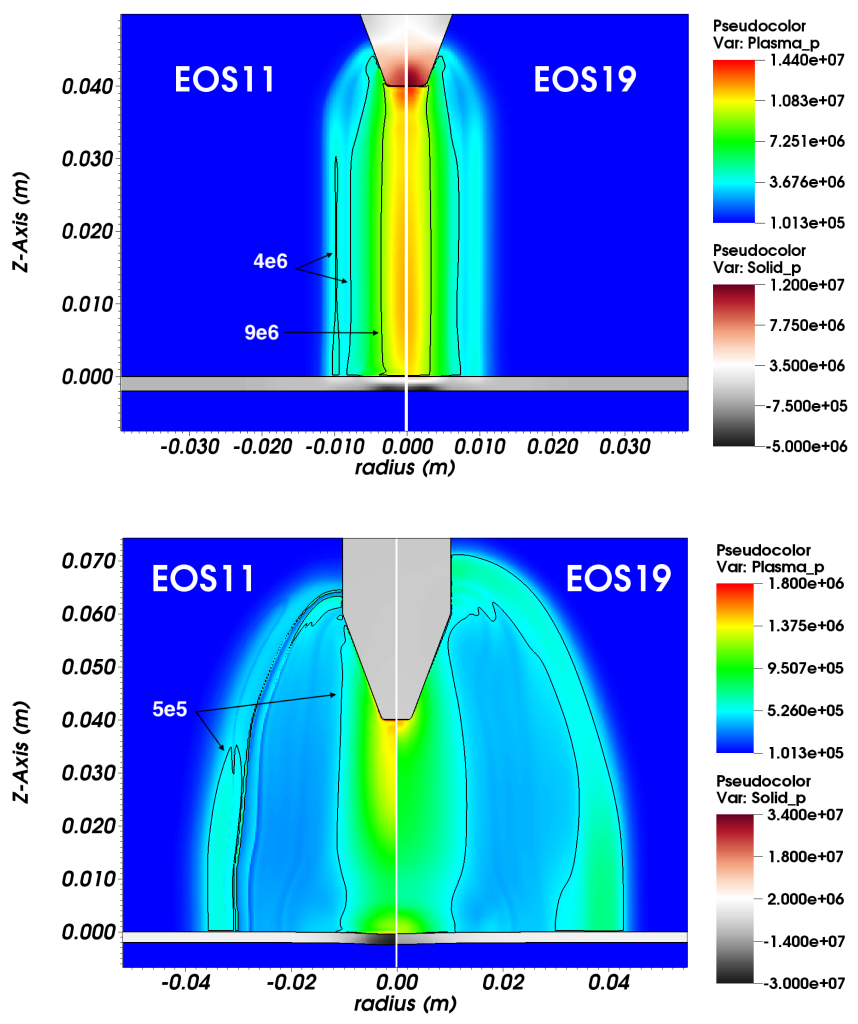

FIG. 7. Pressure distribution and stresses for a component $D$ plasma arc interacting with an aluminium panel. The top image is plotted after $3.18 \mu \mathrm{s}$, and the bottom plot after $25 \mu \mathrm{s}$.

of Martins, where high radiation associated with high temperature would be expected to be visible. Additionally, EOS19 predicts a more uniformly cylindrical shape of the arc channel, including at the attachment point, as is the expected for arc attachment to aluminium 25 .

At $t=25 \mu \mathrm{s}$ the current flowing through the arc has decreased substantially. A characteristic pressure shock wave has emerged, which moves radially outwards, as can be seen in Figure 7. At this time, there are clear differences in the the shock front comparing EOS11 and EOS19. For EOS 11, the shock wave has not travelled as far (approximately $r \approx 4 \mathrm{~cm}$, compared to $r \approx 4.5 \mathrm{~cm}$ for EOS19), and the pressure within the wave is lower. The contour plotted at $P=5 \times 10^{5} \mathrm{~Pa}$ clearly shows the unphysical oscillatory behaviour seen in the plasma discharge results in Figure 4. As expected, EOS19 gives smooth profiles without oscillatory regions throughout the shock wave.

There are similar differences in the temperature profile in Figure 8. For EOS11, the hot spots observed at earlier times are still present, and have expanded in size. These hot spots significantly alter the behaviour of the attachment point to the aluminium substrate, effectively widening the attachment region. For both equations of state, these results also show the aluminium substrate mechanically deforming due to the pressure loading on the panel. 

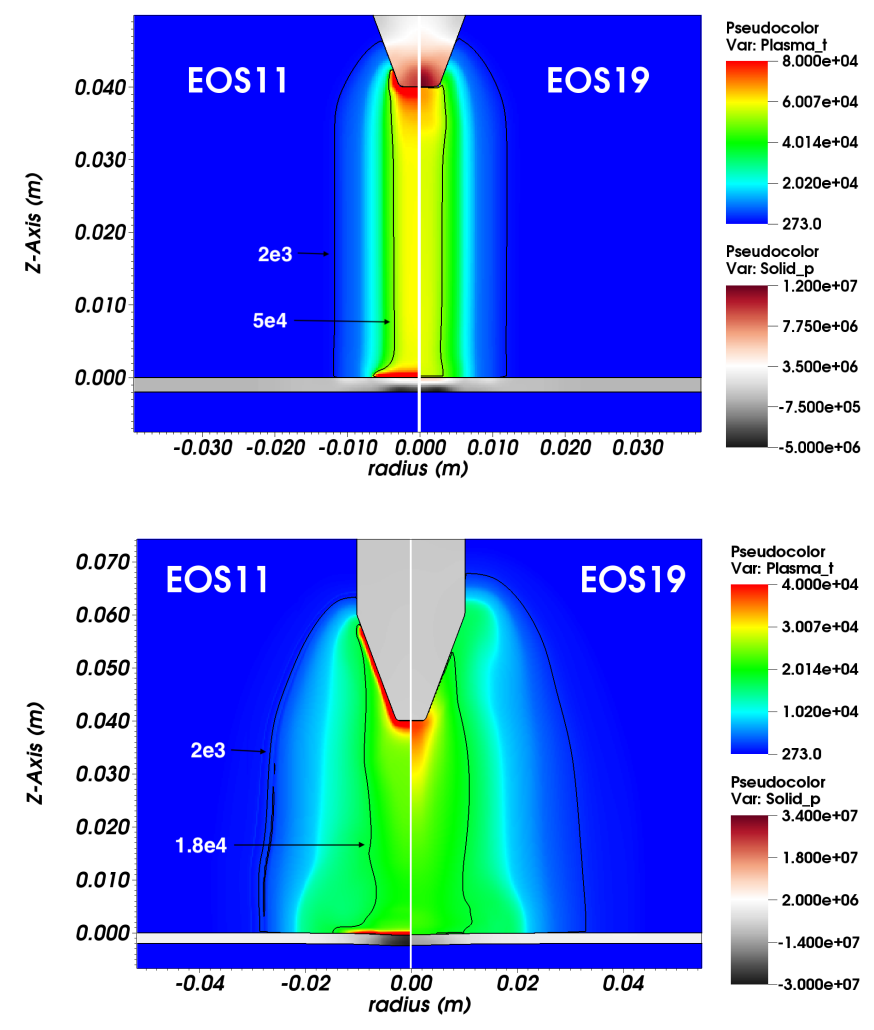

FIG. 8. Temperature distribution and stresses for a component $D$ plasma arc interacting with an aluminium panel. Temperatures at hot spots in EOS11 are substantially higher than $80,000 \mathrm{~K}$. The top image is plotted after $3.18 \mu \mathrm{s}$, and the bottom plot after $25 \mu \mathrm{s}$.

Figure 9 compares the temperature distribution for the two different substrate materials; aluminium and the lowconductivity material. At $t=3.4 \mu \mathrm{s}$, the peak temperature within the arc is similar for both material, around $70,000 \mathrm{~K}$. The arc shape does show small differences, however, the root radius, where it attaches to the substrate, is broader for the low-conductivity material, and this corresponds to a lower temperature in the arc at this point. Figure 10 shows the pressure profiles at corresponding times to the temperature plots in Figure 9. The lower temperature at the attachment point at $t=3.4 \mu$ s corresponds similarly to a lower pressure. Away from the attachment point, the arc profiles are similar for the two substrate materials.

Figures 9 and 10 also show the pressure within the substrate; pressure rise is caused by a direct loading from the arc, but also Joule heating within the substrate. It is clear that the magnitude of the pressure rise is significantly higher for the low conductivity substrate, indicating the vulnerability to greater damage displayed by such materials.

After $t=46 \mu \mathrm{s}$, the pressure shown in Figure 10 shows that the radially expanding shock wave is comparable in structure for both substrates. However, as with the earlier time, at the centre of the arc, pressure is lower directly above the substrate. The temperature plot in Figure 9 shows a significant difference in the arc root structure between the two substrates.
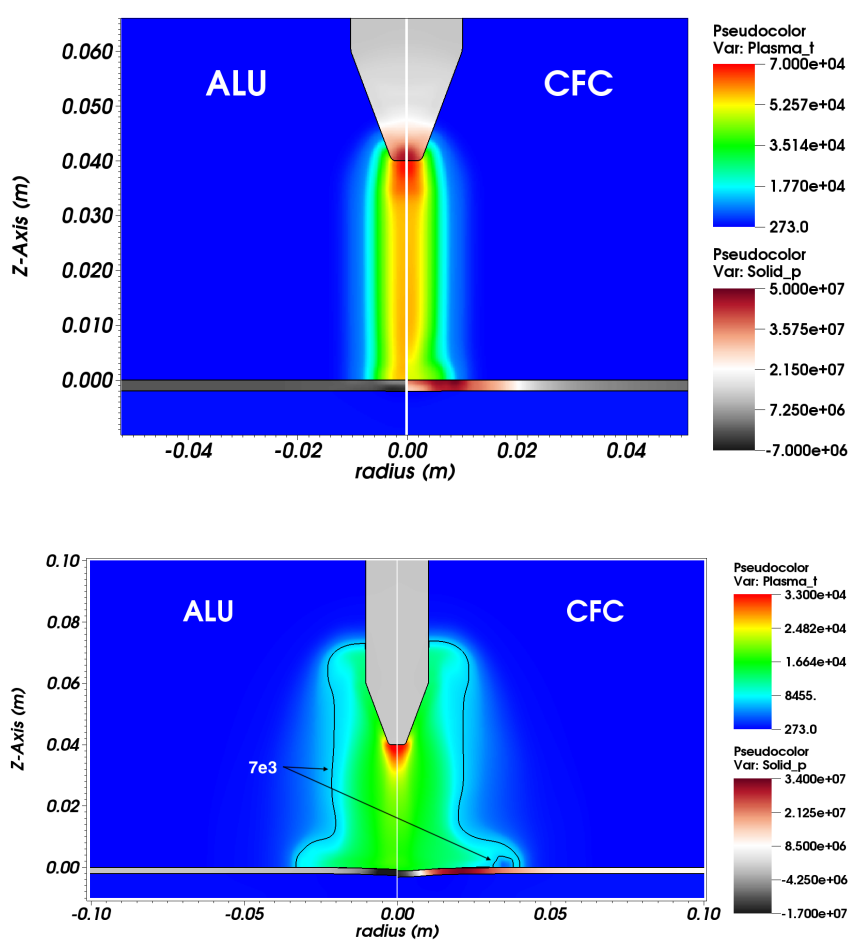

FIG. 9. Temperature distribution and stresses for a component $D$ plasma arc interacting with a carbon composite using EOS19. The top image is plotted after $3.4 \mu \mathrm{s}$, and the bottom plot after $46 \mu \mathrm{s}$.

This shows that for the low conductivity substrate, the arc root is significantly wider at the attachment point. This qualitatively distinct behaviour of the plasma arc root channel between the two substrates is observed experimentally $24 \mid 25$. Of particular interest for the attachment to the low conductivity substrate is the feature which arises in arc root, close to the panel, particularly visible in the temperature profile shown in Figure 9, an emerging filament, which separates from the primary arc channel. The physical mechanism behind this is that the plasma arc provides a favourable path with lower electrical resistance opposed to the direct path to the substrate. Such a filament is a characteristic phenomenon for carbon composite panels, which has been observed in experiments by Martins 24, where the evolution of the visible arc root radius was measured over time for both substrate materials.

The large pressure rise in the low conductivity material is again visible at after $t=46 \mu \mathrm{s}$, and due to the wider arc in this case, the extent of the high pressure region is wider in comparison to the aluminium substrate. Additionally, throughout the evolution, the lower density of the low conductivity material can be seen to result in greater deformation of the panel.

In addition to the qualitative improvement shown by EOS19 for arc attachment simulations, a comparison is made to the measurements of Martins 24 . The expansion of both the arc and the shock wave was measured through optical detection of the attachment process. Figure 11 compares these quan- 

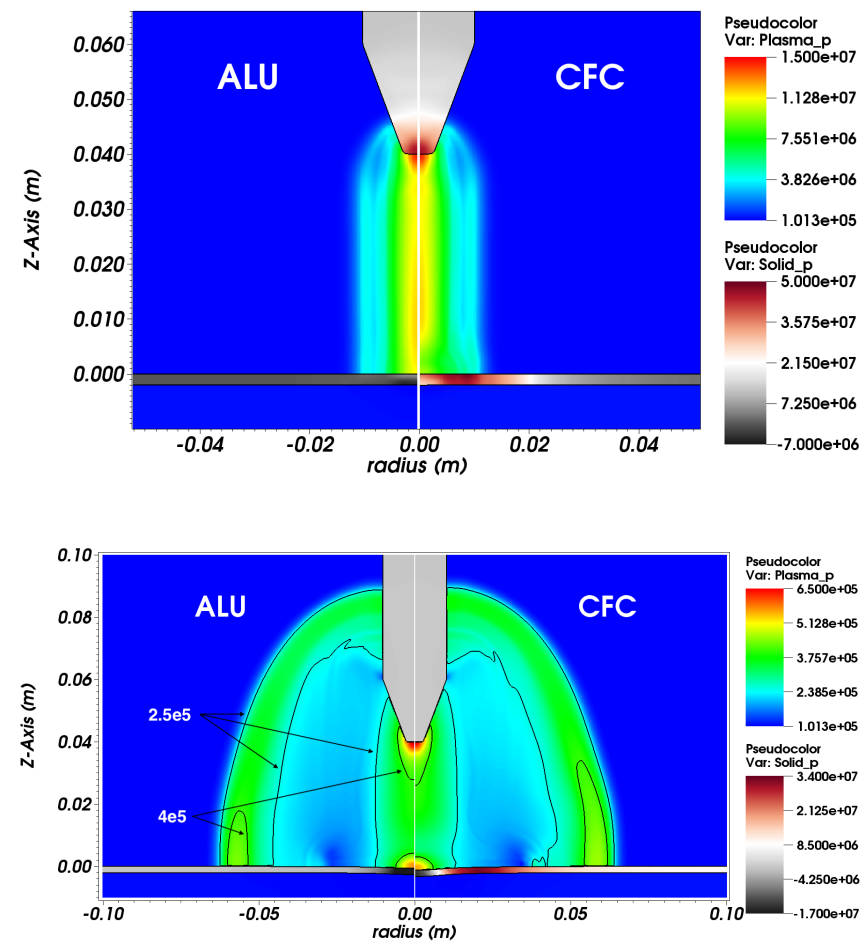

FIG. 10. Pressure distribution and stresses for a component $D$ plasma arc interacting with a carbon composite using EOS19. The top image is plotted after $3.4 \mu \mathrm{s}$, and the bottom plot after $46 \mu \mathrm{s}$.

tities for an aluminium substrate; it is clear that both equations of state follow similar patterns, with EOS19 demonstrating an improvement in accuracy. In order to compare the arc and shock expansion to a low conductivity substrate, following Millmore and Nikiforakis 9 , two measurements are possible; arc growth parallel and perpendicular to the composite weave. The symmetry in these cases makes the cylindrically symmetric model presented here valid. The electrical conductivity used for this comparison is $3 \times 10^{4} \mathrm{~S} / \mathrm{m}$ parallel to the weave and $100 \mathrm{~S} / \mathrm{m}$ perpendicular to it. Results for these tests are shown in Figure 12, where it is noted that in the case of low 'against weave' electrical conductivity in the substrate, EOS11 failed to produce a stable simulation. For the parallel 'with weave' case, results are similar to those in Figure 11. with EOS19 demonstrating an improved fit to the experimental results over EOS11. It is noted that the underprediction in shock radius at early times in the 'against weave' case is due to both the arc itself and the shock being close together, hence it is difficult to measure an accurate radius. At later times, when the two features can be more clearly distinguished, the simulation data matches the experiment well.

\section{CONCLUSIONS}

The primary objective of this work was to implement an improved equation of state for air plasma, which can be used for various applications involving the partial ionisation of air.
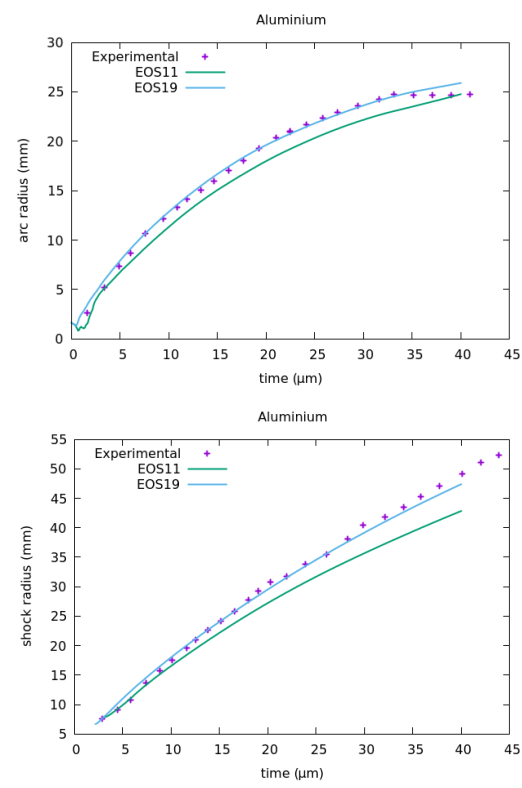

FIG. 11. Comparison of the arc evolution for attachment on aluminium for EOS11 and EOS19. It is clear that EOS19 captures the evolution behaviour well, with the faster expansion of the shock wave demonstrated in Figure 7 matching experimental values. The top plot shows the expansion of the arc radius, whilst the bottom plot shows the shock radius.

In this paper, emphasis was put on the computational multiphysics modelling of solid-plasma interactions. Such numerical tools are of high interest for aeronautics to study lightning strike on new aircraft materials. The equation of state (EOS19) based on the theoretical results of D'Angola et al ${ }^{7 / 8}$, who considered a 19-species air plasma. Their model is based on the Debye-Hückel theory for (partially) ionised gas mixtures, and transport coefficients have been calculated using the Chapman-Enskog theory. Comparison to existing opensource EoSs, e.g. Villa et al $\sqrt[5]{ }$, show EOS19 can accurately capture the evolution of a plasma arc in a non-oscillatory manner. Profiles of various air plasma properties are shown to be accurate over a broad temperature and pressure range. Additionally, the transport coefficients for thermal conductivity have been included in the database for usage in other models in the future.

To validate EOS19, an axisymmetric plasma discharge code was used, simulating a plasma arc channel under laboratory conditions. Results are presented in 1D, based on a model with fixed current density from Villa et al $\stackrel{5}{5}$ and a more realistic model in 2D axisymmetry, which generates the current and magnetic field self-consistently based on the plasma evolution. The results have been validated against experimental and numerical results of others $\sqrt{5|24| 25|38| 39]}$. It was additionally identified that small errors in the levels of ionisation in an equation of state can lead to oscillatory behaviour; the accuracy of EOS19 is demonstrated through the smooth profiles which arise across the temperature and pressure range.

To further test EOS19, it was also applied within a stateof-the-art multimaterial lightning simulation code, which ad- 

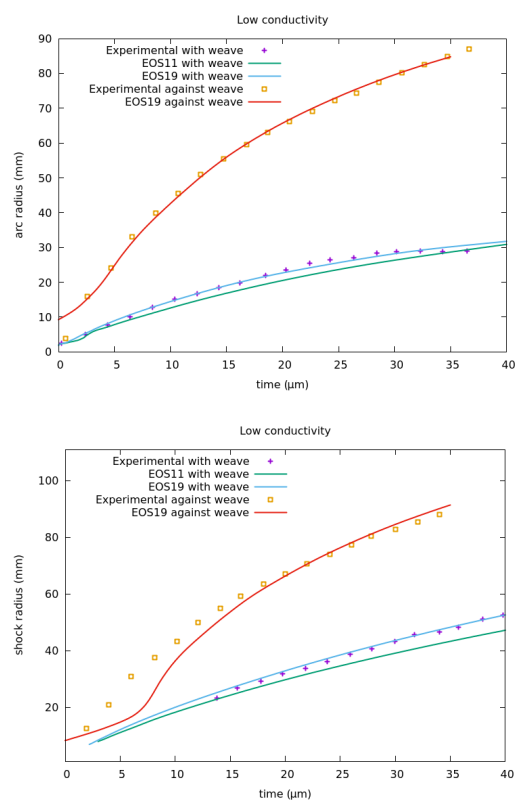

FIG. 12. Comparison of the arc evolution for attachment on the low conductivity substrate for EOS11 and EOS19. Results are shown for two cases, with electrical conductivity corresponding to parallel and perpendicular alignment with a composite weave. In the parallel 'with weave' case, the improvement shown by EOS19 is clear. In the 'against weave' case, EOS11 was unable to simulate this case, whilst EOS19 again shows good agreement. The top plot shows the expansion of the arc radius, whilst the bottom plot shows the shock radius.

ditionally computes the thermomechanical coupling between the plasma arc root and solid aeronautical skin layers. Results are computed for an aluminium and quasi-isotropic CFC panel under extreme lightning testing conditions by inducing a standardised arc current that is used in lightning-protection testing in laboratories. Results for both materials indicate good agreement with experimental measurements by Martins ${ }^{24}$, and no unphysical high-temperature hot spots close to interfaces are observed when applying EOS19. Additionally, a quantitative investigation of the expansion of both the shock wave caused by attachment, and the arc itself, show that EOS19 achieves improved accuracy on EOS11, matching the measured behaviour of these features well.

Future work will focus on improved radiative source term within the arc in a computationally efficient manner. Additionally, one may include possible effects associated with thermal conductivity. Finally, the implementation of a fully $3 \mathrm{D}$ anisotropic equation of state for carbon composite materials will be of high interest for the future development of very complex materials in aeronautics.

To the best of the authors' knowledge, this state-of-the-art lightning code in combination with EOS19 is the only multiphysics code which considers aeronautical materials and their coupled thermomechanical interaction with a plasma arc channel within a single simulation. Hence, the multimaterial lightning code with EOS19 functions as a reliable computational tool to support the design and validation of aeronautical materials concerning lightning protection.

\section{ACKNOWLEDGMENTS}

The authors would also like to thank Micah Goldade of BR\&T, and Carmen Guerra-Garcia of BR\&T and the Massachusetts Institute of Technology, for their input in improving the plasma model.

${ }^{1}$ D. Morgan, C. Hardwick, S. Haigh, and A. Meakins, "The interaction of lightning with aircraft and the challenges of lightning testing," Aerospace Lab Journal 5, 1-10 (2012).

${ }^{2}$ O. Ekici, O. Ezekoye, M. Hall, and R. Matthews, "Thermal and flow fields modeling of fast spark discharges in air," Journal of Fluids Engineering 129, 55-65 (2007).

${ }^{3}$ M. N. Plooster, "Shock waves from line sources. numerical solutions and experimental measurements," The physics of fluids 13, 2665-2675 (1970).

${ }^{4} \mathrm{M}$. Akram and E. Lundgren, "The evolution of spark discharges in gases: I. macroscopic models," Journal of Physics D: Applied Physics 29, 2129 (1996).

${ }^{5}$ A. Villa, R. Malgesini, and L. Barbieri, "A multiscale technique for the validation of a numerical code for predicting the pressure field induced by a high-power spark," Journal of Physics D: Applied Physics 44, 165201 (2011).

${ }^{6}$ L. Mottura, L. Vigevano, and M. Zaccanti, "An evaluation of roe's scheme generalizations for equilibrium real gas flows," Journal of Computational Physics 138, 354-399 (1997).

${ }^{7}$ A. D'angola, G. Colonna, C. Gorse, and M. Capitelli, "Thermodynamic and transport properties in equilibrium air plasmas in a wide pressure and temperature range," The European Physical Journal D 46, 129-150 (2008).

${ }^{8}$ A. D' Angola, G. Colonna, C. Gorse, and M. Capitelli, "Thermodynamic properties of high temperature air in local thermodynamic equilibrium: Ii accurate analytical expression for electron molar fractions," The European Physical Journal D 65, 453-457 (2011).

${ }^{9}$ S. Millmore and N. Nikiforakis, "Multi-physics simulations of lightning strike on elastoplastic substrates," Journal of Computational Physics 405, $109142(2020)$

${ }^{10} \mathrm{M}$. Capitelli, D. Bruno, and A. Laricchiuta, Fundamental aspects of plasma chemical physics: transport, Vol. 74 (Springer Science \& Business Media, 2013).

${ }^{11} \mathrm{P}$. Brimblecombe, Air composition and chemistry (Cambridge University Press, 1996).

${ }^{12}$ W. J. O'Sullivan et al., "Standard atmosphere - tables and data for altitudes to 65,800 feet, report 1235," Tech. Rep. (International Civil Aviation Organization and Langley Aeronautical Laboratory, 1956).

${ }^{13}$ M. Capitelli, G. Colonna, and A. D'Angola, Fundamental aspects of plasma chemical physics: thermodynamics, Vol. 66 (Springer Science \& Business Media, 2011).

${ }^{14} \mathrm{G}$. Colonna and A. D'angola, "A hierarchical approach for fast and accurate equilibrium calculation," Computer physics communications 163, 177-190 (2004).

${ }^{15} \mathrm{G}$. Colonna, "Improvements of hierarchical algorithm for equilibrium calculation," Computer physics communications 177, 493-499 (2007).

${ }^{16}$ J. Haidar, "Non-equilibrium modelling of transferred arcs," Journal of Physics D: Applied Physics 32, 263 (1999).

${ }^{17} \mathrm{~J}$. Hilsenrath and M. Klein, "Tables of thermodynamic properties of air in chemical equilibrium including second virial corrections from 1500K TO 15,000 K,' Tech. Rep. (National Bureau of standards Gaithersburg MD, 1965).

${ }^{18}$ M. Capitelli and E. F. Varracchio, "Thermodynamic properties of Ar-H2 plasmas," Rev. Int. Htes Temp. Et. Refract 14, 195-200 (1977).

${ }^{19}$ R. Sevast'yanov and R. Chernyavskaya, "Virial coefficients of nitrogen, oxygen, and air at temperatures from 75 to $2500 \mathrm{~K}$," Journal of engineering physics 51, 851-854 (1986).

${ }^{20}$ L. D. Landau and E. M. Lifshitz, "Statistical physics," American Journal of Physics 27, 371-372 (1959).

${ }^{21}$ M. Capitelli, G. Colonna, C. Gorse, and A. d'Angola, "Transport properties of high temperature air in local thermodynamic equilibrium," The Eu- 
ropean Physical Journal D-Atomic, Molecular, Optical and Plasma Physics 11, 279-289 (2000).

${ }^{22}$ R. Devoto, "Transport properties of ionized monatomic gases," The Physics of Fluids 9, 1230-1240 (1966).

${ }^{23} \mathrm{~S}$. Chapman and T. Cowling, "“the mathematical theory of non-uniform gases," third edition," Journal Vol 23 (1970).

${ }^{24} \mathrm{R}$. S. Martins, Étude expérimentale et théorique d'un arc de foudre et son interaction avec un matériau aéronautique, Ph.D. thesis, Université ParisSaclay (2016)

${ }^{25} \mathrm{~F}$. Tholin, L. Chemartin, and P. Lalande, "Numerical investigation of the interaction of a lightning and an aeronautic skin during the pulsed arc phase," International Conference on Lightning and Static Electricity (Toulouse, France) (2015).

${ }^{26}$ R. P. Fedkiw, T. Aslam, B. Merriman, and S. Osher, "A non-oscillatory eulerian approach to interfaces in multimaterial flows (the ghost fluid method)," Journal of computational physics 152, 457-492 (1999).

${ }^{27}$ S. K. Sambasivan and H. Udaykumar, "Ghost fluid method for strong shock interactions part 1: Fluid-fluid interfaces,” AIAA Journal 47, 2907-2922 (2009).

${ }^{28}$ S. Schoch, K. Nordin-Bates, and N. Nikiforakis, "An eulerian algorithm for coupled simulations of elastoplastic-solids and condensed-phase explosives," Journal of Computational Physics 252, 163-194 (2013).

${ }^{29}$ L. Michael and N. Nikiforakis, "A multi-physics methodology for the simulation of reactive flow and elastoplastic structural response," Journal of Computational Physics 367, 1-27 (2018).

${ }^{30} \mathrm{~S}$. Godunov and E. Romenskii, "Nonstationary equations of nonlinear elas- ticity theory in eulerian coordinates," Journal of Applied Mechanics and Technical Physics 13, 868-884 (1972).

${ }^{31}$ G. Miller and P. Colella, "A high-order eulerian godunov method for elastic-plastic flow in solids," Journal of computational physics 167, 131176 (2001).

${ }^{32}$ G. Colonna, A. D'Angola, and M. Capitelli, "Electronic excitation and isentropic coefficients of high temperature planetary atmosphere plasmas," Physics of Plasmas 19, 072115 (2012).

33 [Online], "Los Alamos National Laboratory SESAME Database: Equationof-State tabular data for the thermodynamic properties of materials," https://www.lanl.gov/org/padste/adtsc/theoretical/physics-chemistrymaterials/sesame-database.php [Accessed: 2018, July 12].

${ }^{34}$ M. S. Alnæs, J. Blechta, J. Hake, A. Johansson, B. Kehlet, A. Logg, C. Richardson, J. Ring, M. E. Rognes, and G. N. Wells, "The FEniCS project version 1.5," Archive of Numerical Software 3, 9-23 (2015).

${ }^{35}$ E. F. Toro, Riemann solvers and numerical methods for fluid dynamics: $a$ practical introduction (Springer Science \& Business Media, 2013).

${ }^{36} \mathrm{~A}$. Villa, R. Malgesini, and L. Barbieri, "Source Code corresponding to paper: A multiscale technique for the validation of a numerical code for predicting the pressure field induced by a high-power spark," http://gitorious.org/spark2/spark2 [Accessed: 2018, August 14].

37 "Aircraft lightning test methods, ARP5416, SAE," Tech. Rep. (SAE, 2013).

${ }^{38}$ M. N. Plooster, "Numerical model of the return stroke of the lightning discharge," The Physics of Fluids 14, 2124-2133 (1971).

${ }^{39}$ M. N. Plooster, "Numerical simulation of spark discharges in air," The Physics of Fluids 14, 2111-2123 (1971). 